\title{
Open Rupture of Anastomotic Pseudoaneurysm of the Femoral Artery
}

Yu Shomura*, Koji Onoda

Department of Cardiovascular Surgery, Shingu Municipal Medical Center, Wakayama, Japan

\begin{abstract}
A 65-year-old woman presented at our hospital with a skin ulcer and blood oozing from a pulsatile tumor of the left groin. Six years previously she had been treated with an abdominal aorta-left superficial femoral artery bypass using an artificial graft to manage an infected right to left femoro-femoral bypass graft. Computed tomography imaging revealed a ruptured left superficial femoral artery with an anastomotic pseudoaneurysm.

We found during surgery that the original abdominal aorta-left superficial femoral artery bypass graft had become completely detached from the superficial femoral artery. We incised the aneurysm and then resected the hematoma and the ulcerated necrotic skin lesion. The same portion of the artificial graft was resected and partially replaced with the ipsilateral saphenous vein.
\end{abstract}

Keywords: Bypass; Skin ulcer; Saphenous vein

\section{Introduction}

A 65-year-old woman presented at our hospital with a skin ulcer and blood oozing from a pulsatile tumor of the left groin. Six years previously she had been treated with an abdominal aorta-left superficial femoral artery bypass using an artificial graft to manage an infected right to left femoro-femoral bypass graft. She was hemodynamically

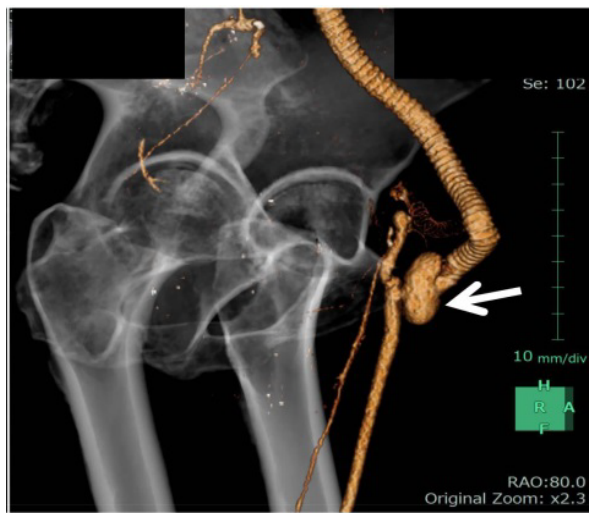

Figure 1: Preoperative findings of enhanced computed tomography. Three-dimensional computed tomography shows graft has completely detached from superficial femoral artery and anastomotic pseudoaneurysm (arrow).

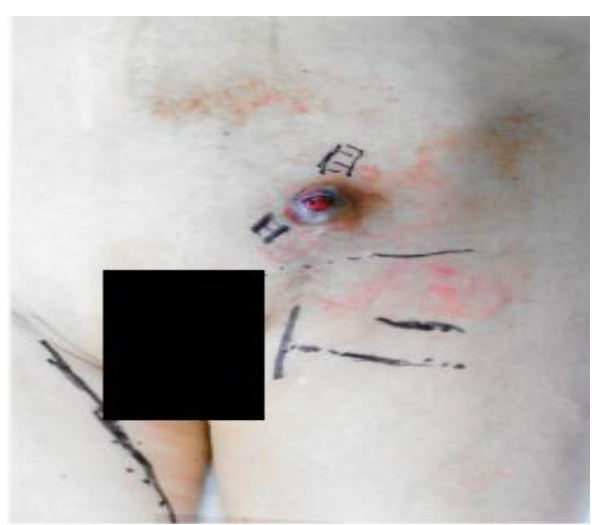

Figure 2: Physical appearance of pseudoaneurysm. stable but had low hemoglobin $(7.6 \mathrm{~g} / \mathrm{dL})$. Computed tomography imaging revealed a ruptured left superficial femoral artery with an anastomotic pseudoaneurysm (Figure 1) that compressed the skin ulcer (Figure 2).

She was surgically treated based on a diagnosis of open rupture of anastomotic pseudoaneurysm of the left superficial femoral artery.

We found during surgery that the original abdominal aortaleft superficial femoral artery bypass graft had become completely detached from the superficial femoral artery, and the intervening tract had dilated to form a pseudoaneurysm. We incised the aneurysm and then resected the hematoma and the ulcerated necrotic skin lesion. The same portion of the artificial graft was resected and partially replaced with the ipsilateral saphenous vein, and a distal anastomosis was

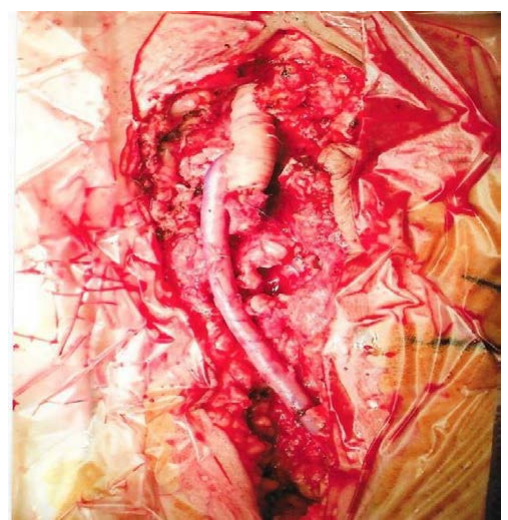

Figure 3: Intraoperative photograph: Saphenous vein graft after replacement.

*Corresponding author: Yu Shomura, Department of Cardiovascular Surgery Shingu Municipal Medical Center, 18-7 Hachibuse, Shingu, Wakayama 647-0072, Japan, Tel: +81-735-31-3333; Fax: +81-735-31-3335; E-mail: shomura82@hotmail.com

Received March 28, 2017; Accepted April 07, 2017; Published April 10, 2017

Citation: Shomura Y, Onoda K (2017) Open Rupture of Anastomotic Pseudoaneurysm of the Femoral Artery. J Vasc Med Surg 5: 305 . doi: $10.4172 / 2329-6925.1000305$

Copyright: (C) 2017 Shomura Y, et al. This is an open-access article distributed under the terms of the Creative Commons Attribution License, which permits unrestricted use, distribution, and reproduction in any medium, provided the original author and source are credited. 
Citation: Shomura Y, Onoda K (2017) Open Rupture of Anastomotic Pseudoaneurysm of the Femoral Artery. J Vasc Med Surg 5: 305. doi: 10.4172/2329-6925.1000305

Page 2 of 2

incorporated into the distal portion of the previous distal anastomosis. We constructed the proximal and distal anastomosis of the saphenous vein graft using the end-to-side technique (Figure 3).

The patient recovered uneventfully and has remained event-free for 11 months.

Anastomotic pseudoaneurysms result from suture line interruption between the graft material and the native vessel. Although all anastomotic sites may be involved, the femoral artery is far more vulnerable with an incidence ranging from $0.5 \%$ to $23.7 \%[1,2]$.

\section{References}

1. Marzo LD, Strandness EL, Schultz RD, Feldhaus RJ (1987) Reoperation for femoral anastomotic false aneurysm. A 15-year experience. Ann Surg 206: 168-172.

2. Klonaris C, Katsargyris A, Vasileiou I, Markatis F, Liapis CD, et al. (2009) Hybrid repair of ruptured infected anastomotic femoral pseudoaneurysms: Emergent stent-graft implantation and secondary surgical debridement. J Vasc Surg 49: 938-945. 